\title{
Special issue on epigenetic inheritance by histone modifications, histone variants and non-coding RNAs
}

\author{
Xiaofeng CAO \\ Institute of Genetics and Developmental Biology, Chinese Academy of Sciences, Beijing 100101, China \\ (C) Higher Education Press and Springer-Verlag Berlin Heidelberg 2011
}

Keeping in view the ever-growing importance of understanding the epigenetic phenomena shaping the behavior of life, our team decided to embark on the idea to organize this special issue of Frontiers in Biology on Epigenetics. Epigenetics refers to the study of heritable changes in gene expression without changes in DNA sequence, which is accomplished by DNA methylation, histone modifications, histone variants, chromatin remodeling, and non-coding RNAs. With the course of time the mechanisms underlying epigenetic regulation are getting inter-connected to each other. This issue comprises eight exciting invited review articles providing a mechanistic and comprehensive insight into different levels of epigenetic inheritance. Majority of the articles highlight the epigenetic regulation by histone modifications, histone variants and non-coding RNAs. This issue of the journal also features a selection of two regular articles, not related to Epigenetics, which proves our devotion to publish all quality articles on a timely basis.

The long-sought-after debates about how histone modification patterns faithfully propagate into daughter cells is reviewed. Hui Wu and Bing Zhu elegantly overviewed two historic models for $\mathrm{H} 3-\mathrm{H} 4$ tetramer spilt or non-spilt decision that govern epigenetic inheritance of histone modifications. The deposition of histone variants to nucleosome adds an additional layer of complexity to the epigenetic regulation. The review article by Leilei Shi and Yuda Fang summarizes the diverse roles played by the incorporation of histone variants into the nucleosome in various biological processes. Wenchao Yin et al. outlined that repeat sequences contribute to centromere formation but are not sufficient to establish and maintain it without epigenetic components. Ying Cao and Ligeng Ma discussed the mechanism of dynamic H2B ubiquitination; the biological functions as well as the molecular mechanisms of H2Bub1. Dosage compensation refers to the equal expression from chromosomes at different copy numbers. James A. Birchler et al. in this special issue described the implications of the gene balance hypothesis for dosage compensation.

Non-coding RNAs are functional RNA molecules that are not translated into proteins including microRNAs, siRNAs and piRNAs and the long ncRNAs etc. Yun Ju Kim and Xuemei Chen reviewed the roles of Mediator, a conserved multiprotein cofactor of RNA Polymerase II in epigenetic regulation via non-coding RNA production. The review by Xiaoyun Jia et al. highlights the key features of newly emerging miRNA-directed DNA methylation in plants. Dacheng Liang et al. presented a model, based on the current knowledge, of the systemic gene silencing in plants mediated by non-coding RNAs.

The current issue does not cover all the aspects of highly diverse field of Epigenetics; however, it does present some of the interesting areas of this rapidly growing field of science. We are convinced that this effort will surely further our current understanding of epigenetic mechanisms involved in countless biological phenomena and will pave the path for the future work to understand many known as well as unknown biological enigmas. We would like to cordially thank all of the authors who generously contributed their knowledge and wisdom to the fulfillment of this effort.

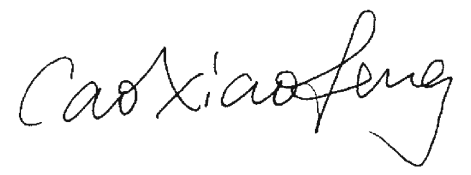

Xiaofeng CAO

Associate Editor-in-Chief

Frontiers in Biology 